\title{
Interplay of phase and glass transitions in crystals
}

\author{
H. Suga \\ Research Institute for Science and Technology, Kinki University, Kowakae 3-4-1, \\ Higashi-Osaka 577, Japan
}

\begin{abstract}
Discovery of glassy crystals indicates that the glass transition occurs widely in condensed phases. In one of glassy crystals, the freezing of disorder occurs before each crystal reaches a hypothetical ordering transition. Examples of these glassy crystals are reviewed in which the prolonged relaxation time necessary for achieving the ordered state prevents the crystal from displaying the whole equilibrium heat capacity associated with the phase transition.
\end{abstract}

\section{INTRODUCTION}

A wide range of substances can be vitrified by cooling their liquids (1). At temperatures well below the fusion point, the relaxation times for viscous flow and thermal equilibrium become so long that the liquid ultimately behaves like a solid (2). These glasses are found to exhibit the glass transition and residual entropy as an indication of non-ergodicity of the frozen disordered system. The relaxation processes of any physical quantities from a non-equilibrium to the equilibrium state are non-linear in nature. The achievement of a unifying theory for relaxation behavior of various kinds of glass-forming liquids is one of the current topics in condensed matter science (3).

Solids can be classified as crystalline and non-crystalline. Glasses are just one example of noncrystalline solids, which can be produced by many methods other than traditional liquid cooling. They are vapor deposition, chemical reaction, mechanical grinding of crystal, pressurization of crystal at low temperatures, and so on (4). We have shown by calorimetric measurements that these exotic noncrystalline solids exhibit likely the glass transition phenomena (5.6). Thus the glass transition is not a characteristic property of liquids but of wide occurrence in condensed matters with some kinds of disorder. In other words, the glass transition is a hindering process that a disordered system undergoes during cooling to reach a more ordered state owing to the prolonged relaxation time for the motion necessary to achieve the ordering (7). It is not unusual during continuous cooling of a disordered system for some aspects of its to fail to maintain thermal equilibrium.

Obviously, the same situation can be expected even in crystalline substances if the crystal possesses some kinds of disorder and the relevant relaxation time increases rapidly at low temperatures. In fact, some mesophases can be undercooled by avoiding transformations into ordered phases, and the undercooled metastable phases ultimately exhibit the glass transition and residual entropy in a manner similar to the undercooled liquids. These freezing processes can also occur in a stable crystalline phase (7). The prolonged relaxation time relevant to the disorder will induce freezing-in process during cooling at a certain temperature interval above or below the possible ordering transition, resulting in important interplay between the phase transition and the glass transition. The present paper reviews several examples where we have observed glass transitions unexpectedly and expectedly in stable and metastable crystalline solids.

\section{EXPERTMENTAL}

\section{Calorimetric spectroscopy}

Adiabatic calorimeters with discontinuous heating mode have long been used to produce the most accurate heat capacity data at low temperatures. Development of the low-temperature calorimetry is closely connected to the experimental verification of the third law of thermodynamics through a comparison between the spectroscopic and calorimetric entropies in an ideal gas state (8). Since two large quantities must be compared in this method, experimental techniques of the adiabatic calorimeter 
have been improved to a highly sophisticated level. The experimental data are manipulated to derive the important thermodynamic functions including the enthalpy, the entropy and the Gibbs energy. The entropy of a system can be related to the disorder each system possesses at high temperatures through the Boltzmann' equation. Good accuracy and precision of the heat capacity data primarily owe to the high thermal stability and temperature resolution of the calorimeter. The measurement is composed of repetition of energizing and equilibration periods and the average heat capacity value is deduced from the ratio of input energy and the resulting temperature rise. The calorimetric temperature is measured as a function of time during each equilibration period. Constancy of the calorimetric temperature with time guarantees the establishment of thermal equilibrium.

If the temperature during the equilibration period changes with time beyond a certain slope that arises from a small amount of residual heat leakage, this means that some irreversible processes are occurring in the sample. If some degrees of freedom of the sample freeze below a narrow temperature interval, systematic temperature drifts can be observed on warming. The relevant frozen-in enthalpy starts to relax toward the equilibrium value and this causes a spontaneous temperature rise of the calorimetric cell. The process requires an extremely long time at low temperatures, so that the next energizing is started before the equilibrium is established. The cycle is repeated in this way until the system apparently crosses the equilibrium state. The subsequent energizing induces the system to deviate from the equilibrium in the opposite direction. Therefore a spontaneous temperature fall can be observed in the subsequent equilibration period. Thus a series of exothermic followed by endothermic processes are observed in a narrow temperature range around the freezing temperature $\left(T_{\mathrm{g}}\right)$. This is just the behavior displayed by glass-forming liquids around their glass transition region.

The same situation occurs in some orientationally disordered crystals. The derived relaxation time data cover the time domain ranging $10^{3}$ and $10^{6} \mathrm{~s}$. Thus the adiabatic calorimeter can be used as an ultralow frequency spectrometer (9) in addition to its conventional use for the determination of various thermodynamic quantities. The method opens a new realm for the study of slow dynamics of molecular motions in condensed state. The time domain covered by this method is complementary with that covered by dielectric spectroscopy.

One important output arising from the improvements of adiabatic control and temperature measurement is a const-ruction of an adiabatic calorimeter for small sample. In the old times, the high quality thermodynamic data were produced at the cost of a large amount of purified sample as large as several ten grams. Such a huge amount of well-characterized sample is not available generally to prepare in many interesting systems, particularly of biological origin. Miniaturization (10) and on-line automation (11) of adiabatic calorimeter were our long dreams. After try and error, we finally succeeded to realize both of them without loss of general accuracy and precision. Nowadays, we can determine accurate thermodynamic quantities with a small amount of sample less than $1 \mathrm{~g} \mathrm{(12)}$ by using a special temperature transfer system in which a thermometer is removed from a sample cell so as to minimize its heat capacity. The calorimetric temperature is transferred to a copper block by using a fine thermocouple and the temperature of the block is measured with a platinum resistance thermometer based on the International Temperature Scale by standard method. A typical calorimetric cell for a sample of small mass $(2 \sim 3 \mathrm{~g})$ is shown in Fig. 1.
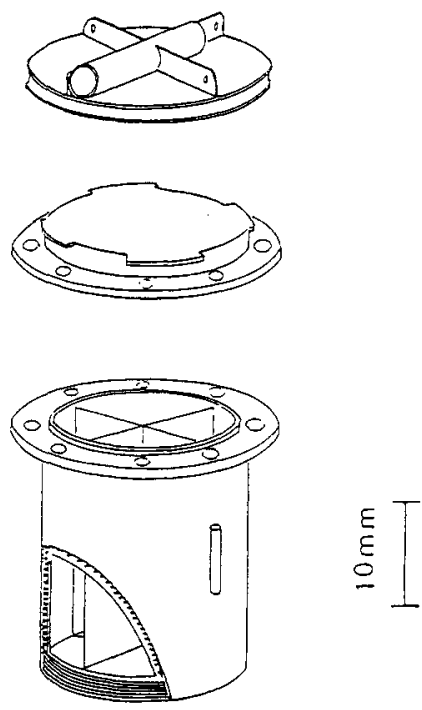

\section{Dielectric spectroscopy}

Dielectric relaxation is closely related with the freezing process of dipolar molecules or ions in any disordered systems. Many polar liquids are known to exhibit the dielectric relaxations in their undercooled states and the dielectric relaxation time at the glass transition $T_{\mathrm{g}}$ becomes of the order of $1 \mathrm{ks}$ which corresponds to the time scale of a single heat-capacity determination. There are many crystals which also exhibit the dielectric relaxations. Therefore, it is necessary to apply the dielectric spectroscopy in clarifying the relation between the phase transition and freezing process in crystals composed of dipolar molecules or ions. Real and imaginary parts of dielectric permittivity were 
measured by a capacitance bridge (General Radio 1615A) in the frequency range 20 100 $\mathrm{Hz}$ and by an LCR meter (Yokogawa Hewlett Packard 4284A) in the range $100 \mathrm{~Hz} 1 \mathrm{MHz}$. A home-made cryostat was used for the temperature change of samples.

\section{RESULTS AND DISCUSSION}

\section{Freezing of head-to-tail orientational disorder}

\section{A. Alkali cyanides}

Both $\mathrm{NaCN}$ and $\mathrm{KCN}$ crystals exhibit trimorphs. At room temperature, they crystallize into a cubic system with the NaCl-type structure. The dumbbell-shaped $\mathrm{CN}^{-}$ion has eight equi-energetical orientations along the [111] and corresponding axes in the unit cell, giving rise to time- and spaceaveraged cubic symmetry. The orientational disorder can be removed by two-step ordering processes $(13,14)$. On cooling, the crystal undergoes a first-order transition at $287 \mathrm{~K}$ for $\mathrm{NaCN}$ and at $168 \mathrm{~K}$ for $\mathrm{KCN}$ to transform into an orthorhombic system. An elastic constant $c_{44}$ of $\mathrm{KCN}$ crystal was found to decrease (15) dramatically with decreasing temperature, in contrast to normal crystals. The softening effect induces an elastic instability of the cubic phase and the transformation saves the mechanical crisis. X-ray diffraction analysis showed that the $\mathrm{CN}$ ions align along the $\mathrm{b}$ axis of the orthorhombic cell. Consideration of the associated entropy change suggests that the head-to-tail disorder of the $\mathrm{CN}^{-}$ ion still exists in this phase. The remaining ordering driven by the electrostatic dipole-dipole interaction among the anions was found to occur at $172 \mathrm{~K}$ for $\mathrm{NaCN}$ and $83 \mathrm{~K}$ for $\mathrm{KCN}$, resulting in establishment of full order of the $\mathrm{CN}^{-}$ions in an antiferroelectrical way (16). Similar ordering transitions were naturally expected for $\mathrm{RbCN}$, which has the same cubic system at room temperature and a larger cationic radius. When we measured the heat capacity of RbCN in 1968 (17), we could only observe the elastic transition with a long tail of excess heat capacity in the low temperature side. If we plot the transition temperature against the inter-anionic distance as shown in Fig. 2 , the corresponding electric transition was expected to appear at around $40 \mathrm{~K}$ in $\mathrm{RbCN}$. A simple calculation of the potential hindering the head-to-tail motion suggested that a rather high potential barrier would result in a freezing out of the motion before the crystal reaches the expected transition.

Almost twenty years later when the adiabatic control of a calorimetric system was highly improved and a melt-grown single crystal of $\mathrm{RbCN}$ was available, the heat capacity was measured again (18). The experimental results are shown in Fig. 3. The total heat capacity is composed of the lattice-vibrational contributions and excess part due to the ordering processes. The excess heat capacity is drawn in Fig. 4.

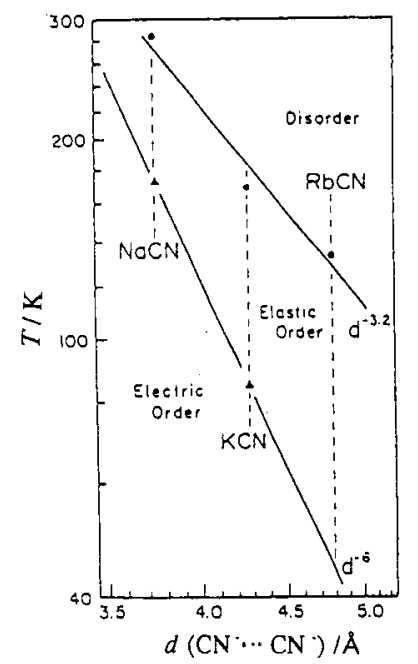

Fig. $2 T_{\mathrm{trs}} \sim d\left(\mathrm{CN}^{\top} \cdots \mathrm{CN}\right)$ plot of alkali cyanides.

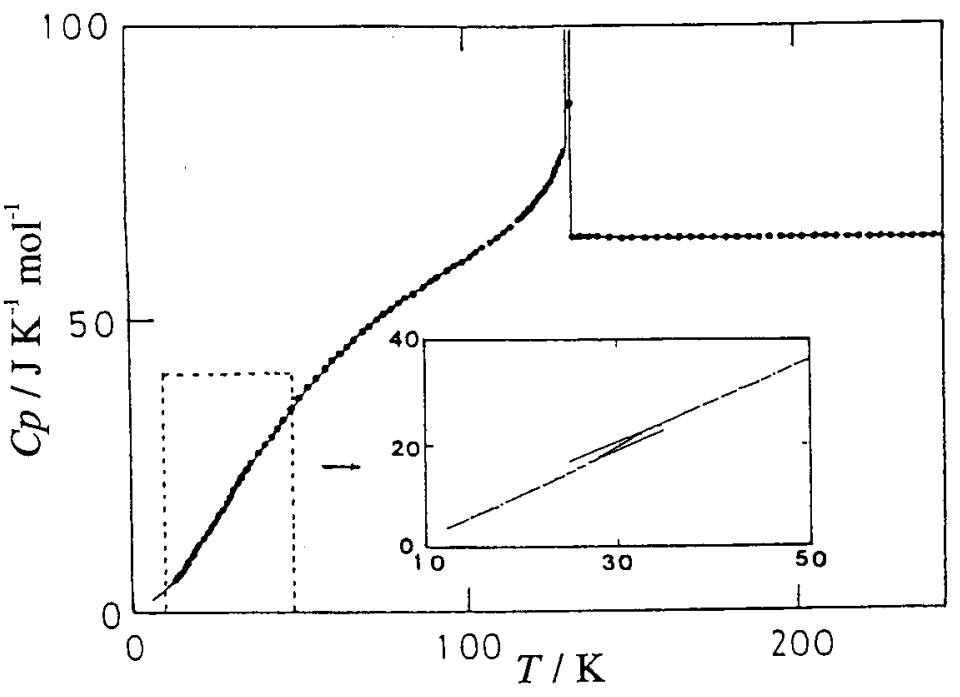

Fig. 3 Molar heat capacity of RbCN crystal. 


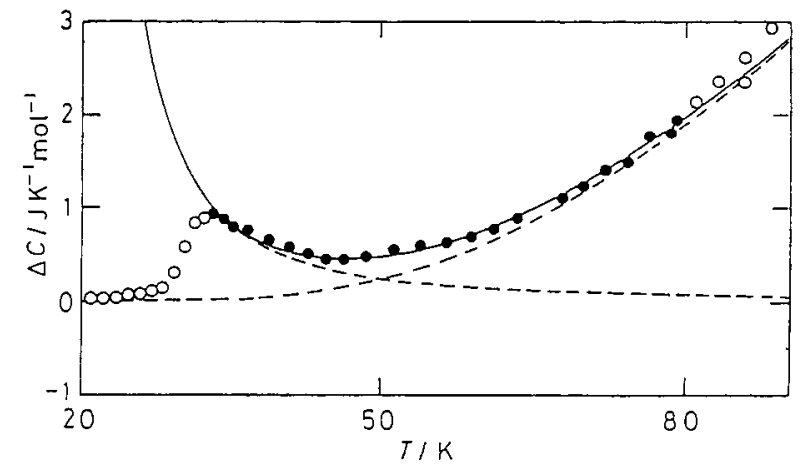

$S=1 / 2$ Ising model of the fcc lattice

$$
\begin{array}{cc}
T>T_{\mathrm{c}} & w=\tanh \left(J_{1} / R T\right) \\
C / R= & \left(J_{1} / R T\right)^{2}\left(6+48 w+390 w^{2}+3216 w^{3}\right. \\
& \left.+26844 w^{4}+229584 w^{5}\right) \\
T<T_{\mathrm{c}} & u=\exp ^{3}\left(-4 J_{2} / R T\right) \\
C / R= & \left(J_{2} / R T\right)^{2}\left(576 u^{6}+1161 u^{11}-14976 u^{12}\right. \\
& \left.+28800 u^{15}+172032 u^{16}\right) \\
& \\
J_{1}=20.8 \mathrm{~J} \mathrm{~mol} l^{-1} \\
T_{\mathrm{c}}=24.5 \mathrm{~K}
\end{array}
$$

Fig. 4 Excess heat capacity of $\mathrm{RbCN}$.

The figure clearly indicates an evidence of glass transition. Exothermic followed by endothermic spontaneous temperature drifts were observed in a systematic way at temperatures around $30 \mathrm{~K}$. Since the $\mathrm{CN}^{\top}$ ion carries a small dipole moment, the freezing process reveals itself as the dielectric relaxation. The enthalpy relaxation rate observed calorimetrically could be analyzed by a function consisting of terms exponential and linear in time. The latter term is a correction for a small amount of residual heat leakage. The enthalpy relaxation time $\tau$ data thus derived could be well correlated with those obtained by NMR, dielectric and other measurements in an Arrhenius plot covering $\tau(T)$ over 12 decades from $10^{9}$ to $10^{-3} \mathrm{~s}(19)$. The relaxation time at $30 \mathrm{~K}$ becomes of the order of $10^{3} \mathrm{~s}$, which is the time scale for a single heat-capacity determination. Thus the prolonged relaxation time for the flipflop motion of $\mathrm{CN}^{-}$ions in $\mathrm{RbCN}$ crystal hinders the observation of the possible ordering process below that temperature in the time window of the calorimetric measurement.

The excess heat capacity of RbCN given in Fig. 4 can be resolved into two components. One is the low-temperature tail of the elastic transition and the other the high-temperature tail of the hypothetical electric transition. Since the head-to-tail disorder of the $\mathrm{CN}^{-}$ion has a magnetic analogy of the Ising system with spin $1 / 2$ (up or down), the problem can be treated by using theoretical equations for the magnetic transition. Statistical mechanics of magnetic systems gives mathematical expressions for the excess heat capacity in terms of a pair interaction energy $J$ for the Ising models (20).

Series expansion methods for the $S=1 / 2$ Ising system of an fcc lattice give analytical equations and are shown in the right space of Fig. 4 for $T>T_{\mathrm{c}}$ and $T<T_{\mathrm{c}}$, respectively, where $T_{\mathrm{c}}$ is the transition temperature. In this way, the excess heat capacity between the glass transition and elastic transition can be reproduced by a sum of the two contributions with parameters $J_{1}$ and $J_{2}$. Here, $J_{1}$ characterizes the hypothetical electric transition and $J_{2}$ the elastic transition. The best-fit values of the interaction parameters are $J_{1}=(20.8 \pm 0.2) \mathrm{J} \mathrm{mol}^{-1}$ and $J_{2}=(132.5 \pm 0.5) \mathrm{J} \mathrm{mol}^{-1}$. The reproduced heat capacity curves are also drawn in Fig. 4. The parameter $J_{1}$ immediately gives the hypothetical ordering temperature as $24.5 \mathrm{~K}$. Extrapolation of the relaxation time data to this temperature suggests that we require about $4.6 \times 10^{6} \mathrm{~s}$ (53 days) in order to realize the fully ordered state in RbCN crystal. At the moment, it is not practical to hold a RbCN sample at a cryogenic temperature for a long time.

\section{B. Carbon monoxide}

A classic example of the freezing of head-to-tail disorder can be found in $\alpha-\mathrm{CO}$ crystal. The reorienting moments slow down in a thermally activated process in the crystalline field arising from the neighboring molecules. The residual entropy of $4.4 \mathrm{~J} \mathrm{~K}^{-1} \mathrm{~mol}^{-1}(\sim R \ln 2)(21)$ reported by Clayton et al.was interpreted in this way. Many textbooks describe that the $\mathrm{CO}$ molecule carries a small dipole moment and the head-to-tail disorder is frozen well above the hypothetical transition temperature, estimated to be $5 \mathrm{~K}(24)$, because of the high potential barrier hindering the reorientational process. Later heat capacity measurements $(22,23)$ found no indication of possible phase transition below $14 \mathrm{~K}$, the lowest temperature covered by Clayton and Giauque. Nobody knows the temperature at which the freezing actually took place. Curl et al (25) discussed the kinetics of the ordering in CO crystal from a quantum mechanical point of view. Upon closer examination of the literature, however, we found a small deviation of the $C_{p}$ data at about $18 \mathrm{~K}$ from a smoothed curve in $C_{p} / T^{-3}$ versus $T$ plot. This plot greatly tempers the heat capacity rapidly changing with temperature to show any small anomalous behavior in a magnified scale. A glass transition due to freezing was conjectured and this recognition 


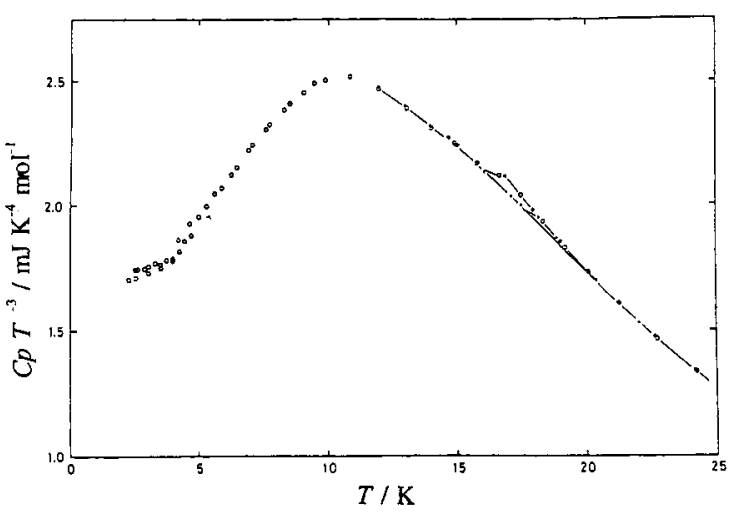

Fig. 5 Heat capacity of $\mathrm{CO}\left(C_{p} T^{-3}\right)$.

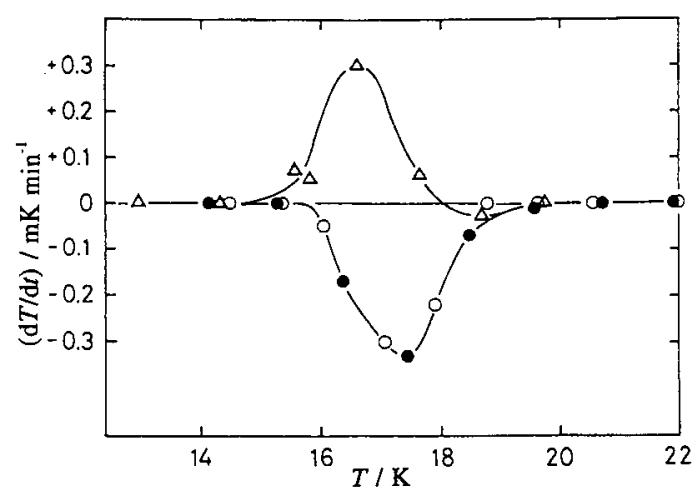

Fig. 6 Spontaneous temperature drifts of $\mathrm{CO}$.

motivated us to remeasure the heat capacity of $\mathrm{CO}$ below $20 \mathrm{~K}$, focusing attention on the annealing effect of the anomalous heat capacity (26).

Figure 5 shows the new experimental data in the form of $C_{p} / T^{-3}$ plotted against temperature. The small anomaly observed in the sample normally cooled (series 1) was increased by annealing the sample at $16 \mathrm{~K}$ for $19 \mathrm{~h}$ (series 2) or at $15 \mathrm{~K}$ for $39 \mathrm{~h}$ (series 3). The average temperature drift rate (dT/d $t$ ) observed during the initial $20 \mathrm{~min}$ in each equilibration period is plotted in Fig. 6 as a function of temperature for the three series of measurements. The data for series $1(\Delta)$ exhibit exothermic followed by endothermic spontaneous changes of calorimetric temperature around $17 \mathrm{~K}$, while the annealed samples ( $O$ and $\bullet$ ) show only endothermic temperature drifts. These are typical behaviors of the spontaneous temperature drifts observed in glass-forming liquids around their $T_{\mathrm{g}}$ and in ordinary ice around $100 \mathrm{~K}$. Thus it can be concluded that the flip-flop motion of the $\mathrm{CO}$ molecules freezes out at temperature around $18 \mathrm{~K}$, at which the short-range order starts to develop. Annealing tends to relax the sample toward more ordered state with less entropy. Upon reheating, the annealed sample acquires an entropy change greater than that of the unannealed sample, resulting in absorption of extra energy from the lattice heat reservoir. These relaxation processes continue up to $20 \mathrm{~K}$, above which the thermal equilibrium is attained rapidly. The apparently rapid equilibration below $15 \mathrm{~K}$ is of quasiequilibrium nature due to extremely prolonged relaxation times.

\section{Freezing of positional disorder coupled to orientational disorder}

\section{A. Alkali nitrites}

Alkali nitrites also crystallize into a cubic system with the CsCl-type structure. The V-shaped nitrite ions are orientationally disordered at room temperature in conformity with the octahedral symmetry of the anion sites. The orientational disorder can be removed by a phase transition on cooling (27). For example, $\mathrm{CsNO}_{2}$ transforms at $209 \mathrm{~K}$ into a rhombohedral system. Consideration of the packing of the $\mathrm{NO}_{2}{ }^{-}$ion inside the rhombohedral unit cell suggests that the principal axis of the ion (perpendicular to the dipole moment of $\mathrm{NO}_{2}{ }^{-}$) should orient along the 3-fold symmetry axis of the cell. This means that the $\mathrm{NO}_{2}{ }^{-}$ion has three equi-energetical orientations in the unit cell and the reorientational motion must persist in the low temperature phase in order to fulfill the site symmetry. The remaining disorder should be removed by another ordering transition, as in the cases of $\mathrm{NaCN}$ and $\mathrm{KCN}$. Actually, however, the situation is similar to the case of RbCN. The heat capacity exhibits a glass transition at around $42 \mathrm{~K}$, as shown in Fig. 7. Since the $\mathrm{NO}_{2}{ }^{-}$ion carries a fairly large dipole moment, freezing out of the reorientational motion can be detected by dielectric measurement. The dielectric permittivity and the corresponding loss measured at various frequencies ranging $100 \mathrm{~Hz}$ and $100 \mathrm{kHz}$ are shown in Fig. 8 as a function of temperature in a relevant region.

As has been described earlier, the spontaneous temperature change can be analyzed by assuming a linear relaxation process. The relaxation time data thus calculated are plotted as a function of reciprocal temperature in Fig. 9 for $\mathrm{CsNO}_{2}$ and in Fig. 10 for $\mathrm{TINO}_{2}$, respectively. The latter crystal exhibits essentially the same behavior and the corresponding glass transition appears at around $60 \mathrm{~K}$. The difference in $T_{\mathrm{g}}$ may arise from the differences in the crystal structures (orthorhombic for $\mathrm{TINO}_{2}$ ) and in the ionic radii of both cations. The larger ionic radius of $\mathrm{Cs}^{+}$ion $(169 \mathrm{pm})$ compared with that of $\mathrm{Tl}^{+}$ion (144 pm) will provide a space which enhances the orientational mobility of anion in the low temperature phase. It is worthy of note that the relaxation time data derived from the dielectric and 
calorimetric spectroscopies lie on the same straight line over a wide range of time. Again, both techniques have observed the same motion with different time windows, indicating the complementarity of the two experimental methods.

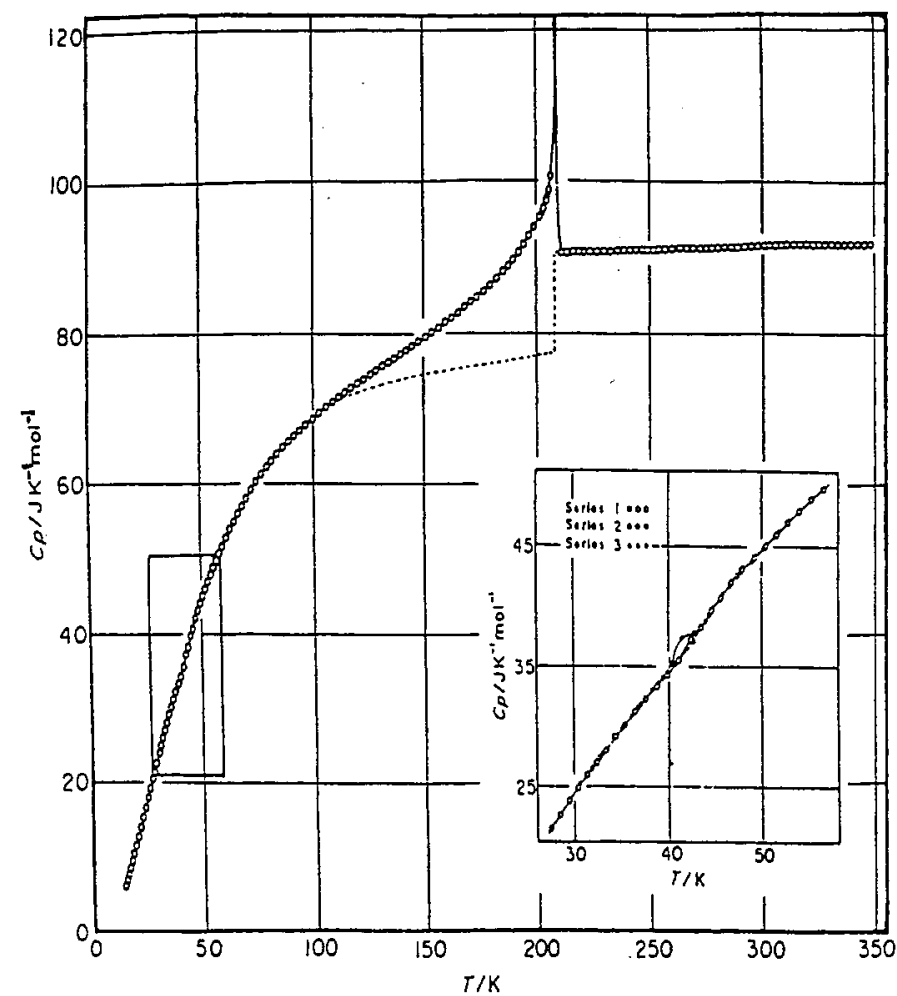

Fig. 7 Heat capacity of $\mathrm{CsNO}_{2}$.
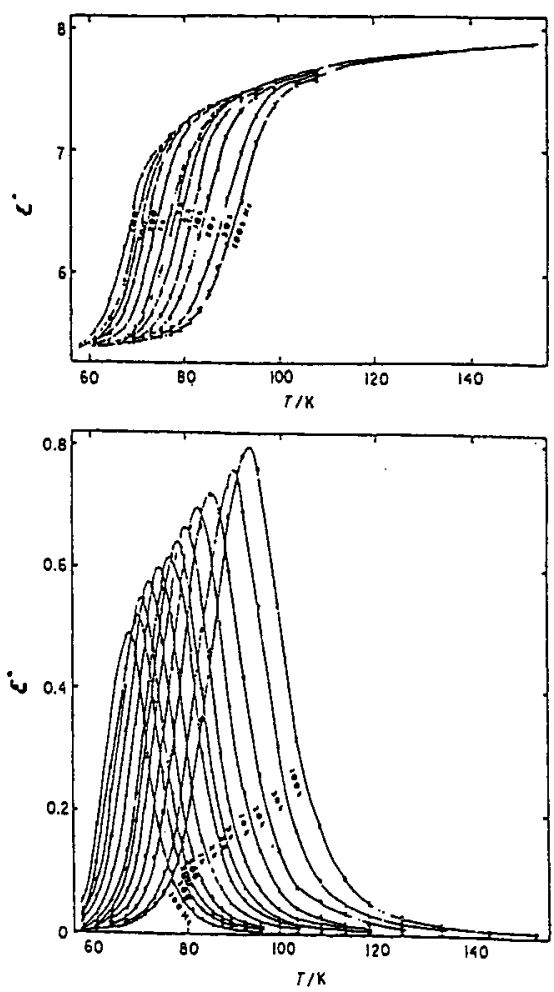

Fig. 8 Dielectric relaxation of $\mathrm{CsNO}_{2}$.

Formation of a solid solution was found between $\mathrm{CsNO}_{2}$ and $\mathrm{TINO}_{2}$ over the whole concentration range at high temperatures (28). This behavior can be observed rather commonly in organic plastic crystals (29). Increased disorder generally enhances the mutual solubility. Therefore it might be intriguing to examine what will happen if the solid solution is cooled down. Five specimens of solid solution of the binary system $\left(\mathrm{CsNO}_{2}\right)_{\mathrm{x}}\left(\mathrm{TINO}_{2}\right)_{1-\mathrm{x}}$ were prepared by slow evaporation of solutions containing appropriate amounts of the component crystals. They were well annealed for homogenization of composition at some temperatures below the melting point. The annealing process was continued for about $300 \mathrm{~h}$ until their X-ray powder diffraction gave sharp patterns comparable to those of pure component systems (27).

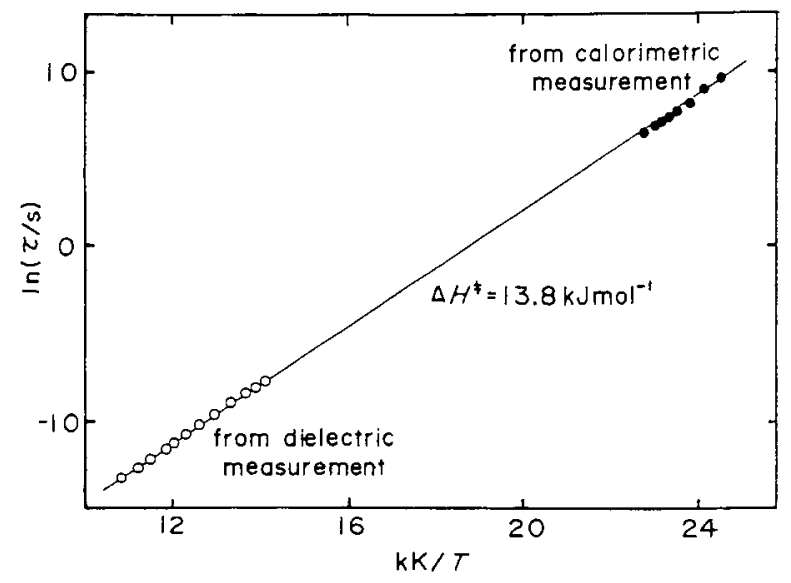

Fig. 9 Relaxation time of $\mathrm{CsNO}_{2}$.

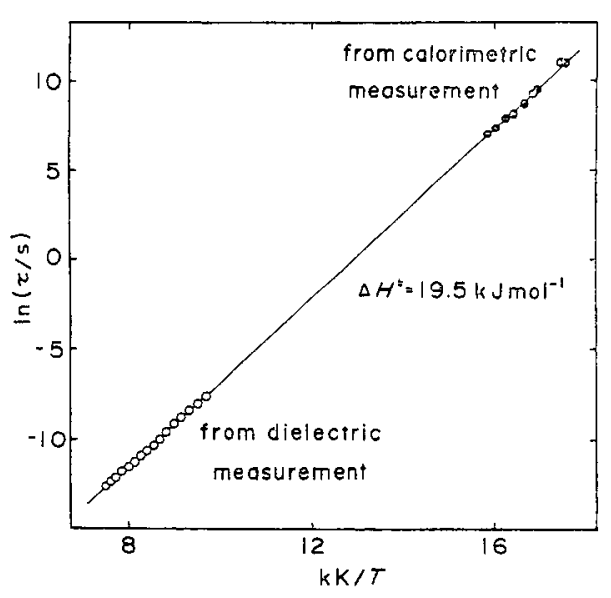

Fig. 10 Relaxation time of $\mathrm{TlNO}_{2}$. 
All the specimens undergo the corresponding ordering and glass transitions. The temperatures at which the transitions occur depend on the composition. Sharpness of the heat capacity peak associated with the phase transition supports enough homogenization of the composition in the solid solution. The glass transition is, however, broader and appears at a temperature between those of the pure crystals. Both of the relaxation times for the enthalpy and the dielectric polarization related to the freezing have wider distributions than those of each neat crystal, indicating enriched environments at anion sites arising from various combinations of both cations. At temperatures in the range between 203 and 212 $\mathrm{K}$, all the specimens showed exothermic effects. The phenomena elapsed over a long period of time $(\sim 300 \mathrm{~h})$, depending on the composition and temperature. The low temperature behavior of the wellannealed sample greatly differed from that of the unannealed sample. Figure 11 draws the heat capacity of a sample $\mathrm{Cs}_{0.695} \mathrm{Tl}_{0.305} \mathrm{NO}_{2}$ in both states. The annealed sample exhibits a higher-order transition with peak temperature at $240 \mathrm{~K}$ in addition to a first-order transition at $212 \mathrm{~K}$. Furthermore, two glass transitions appear at temperatures which correspond approximately to those of $\mathrm{CsNO}_{2}$ and $\mathrm{TINO}_{2}$. This means that the slow exothermic process arises from a phase separation of the solid solution into the pure components. On warming, the annealed sample composed of a mixture of two crystals exhibits the first-order transition at the eutectoid temperature of $212 \mathrm{~K}$. The increased orientational disorder of the anion enhances the mutual miscibility of both crystals to recover the original uniform solid solution. The higher-order transition just corresponds to the recovering process. Actually, the entropy change associated with the higher-order transition is almost equal to the ideal mixing entropy with corresponding composition, $-R[x \ln x+(1-x) \ln (1-x)]$. The phase diagram for the metastable and stable sequences is shown in Fig. 12.

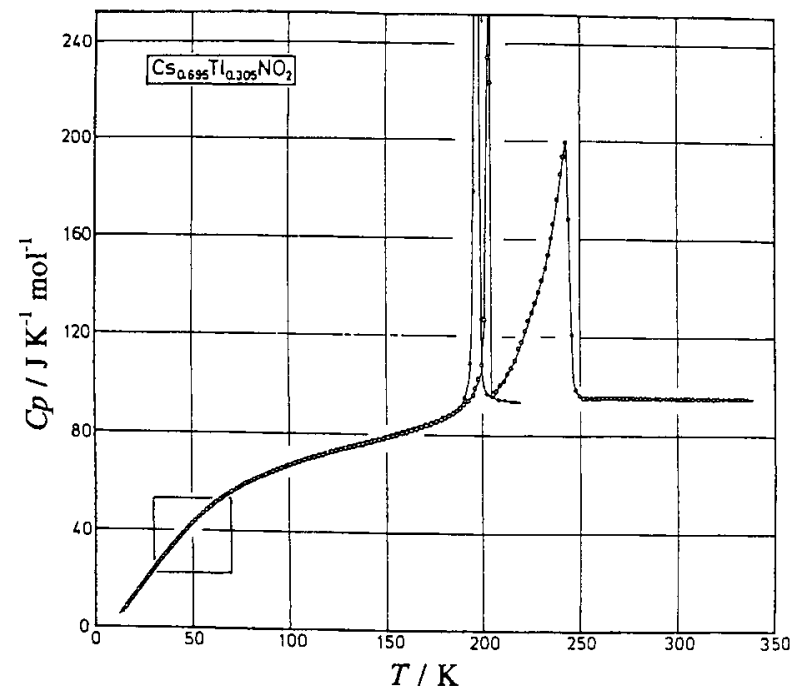

Fig. 11 Heat capacity of $\mathrm{Cs}_{0.695} \mathrm{Tl}_{0.305} \mathrm{NO}_{2}$.

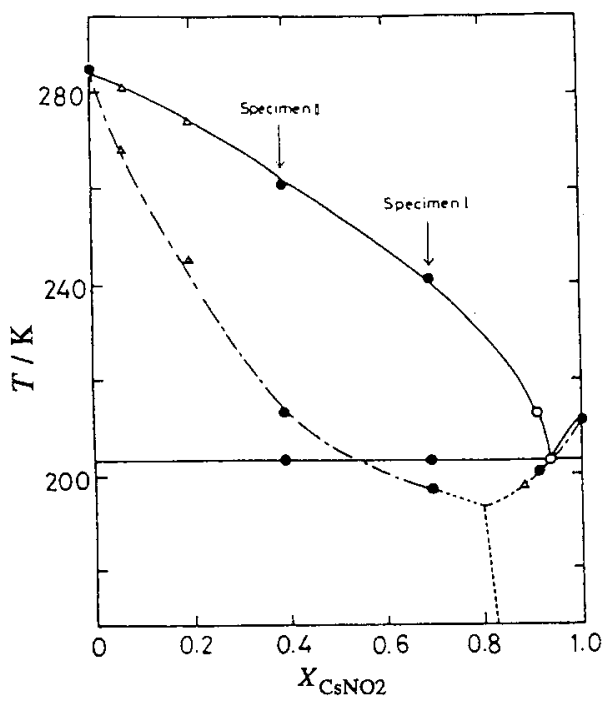

Fig. 12 Phase diagram of $\mathrm{Cs}_{\mathrm{x}} \mathrm{Tl}_{1-\mathrm{x}} \mathrm{NO}_{2}$ system. - stable phase; - - metastable phase

It turns out that the normally cooled sample has double frozen-in disorder in the low temperature phase with respect to the positional degrees of freedom of the cations coupled to the orientational degrees of freedom of the anions. The positional disorder can be removed by annealing the sample at temperatures below the eutectoid point for a long time. Phase separation is one pattern of the positional ordering. The separated pure crystals recover their orientational mobility above the eutectoid temperature and rebuild the uniform solution. In this case, the associated entropy of mixing can be observed directly by the calorimetry. Generally, phase separation cannot be attained at low temperatures within a reasonable time owing to slowing down of the diffusional process.

\section{B. Camphor}

D,L-camphor shows similar behavior at low temperatures. Optical enantiomers, D- and L-camphor, form a complete solid solution at room temperature (phase II). The low symmetry of the chiral molecule indicates that the camphor is one orientationally disordered crystal. D-camphor transforms at $244.6 \mathrm{~K}$ into a tetragonal phase (III) through a first-order transition, while D,L-camphor exhibits a higher order transition at $204 \mathrm{~K}$. Schäfer et al .(30) concluded from thermodynamic measurement that 
both $\mathrm{D}$ - and $\mathrm{D}, \mathrm{L}$-camphor have their ordered lattices at $0 \mathrm{~K}$, in accord with the third law of thermodynamics. However, there are significant differences in their transition behavior. An X-ray study showed that the diffraction pattern clearly changed for D-camphor at $244.6 \mathrm{~K}$ transition, whereas there is little change for D,L-camphor at $204 \mathrm{~K}$ (31). The dielectric permittivity (32) of the latter shows a relaxation process at low temperatures. These observations suggest some disorder still persisting in the phase III of D,L-camphor, in contrast to D-camphor. This contradiction motivated us to remeasure the heat capacities of D- and racemic camphors around their III-II transitions (33).

Actually a preliminary DTA measurement showed that the transition temperature shifted unbelievably when a D,L-camphor sample was annealed at temperatures just below $204 \mathrm{~K}$. The transition temperature moved to $215 \mathrm{~K}$ if the sample was annealed at around $202 \mathrm{~K}$ for $13 \mathrm{~h}$. This observation shows that something obviously happened during the annealing process. The $C_{p}$ data of $\mathrm{D}$-camphor and $D, L$-camphor are summarized in Fig. 13. The sample cooled with the rate of $-1 \mathrm{~K} \mathrm{~min}^{-1}$ is denoted by subscript $r$. The annealing of the sample (designated as suffix a) was carried out using a series of

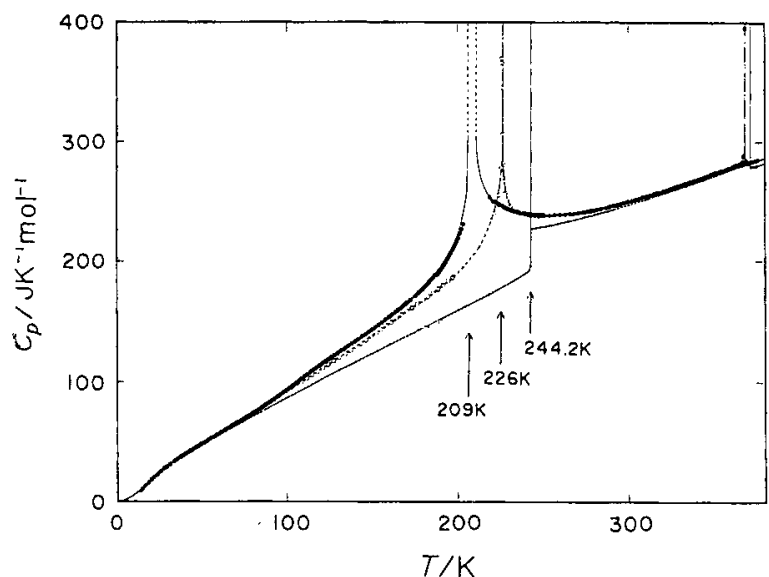

Fig. 13 Heat capacity of camphor. - sample r; O sample a

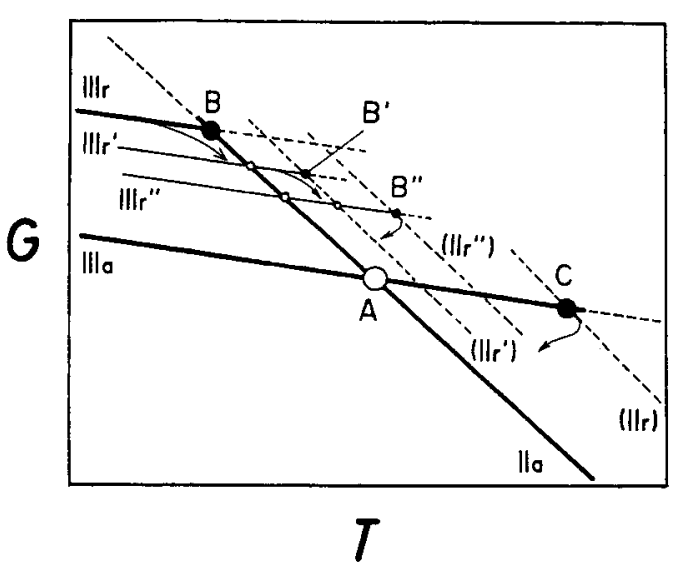

Fig. 14 Schematic diagram of the Gibbs energy of D,L-camphor (phases II and III). (Units of $G$ and $T$ are arbitrary)

short-interval temperature rise $\Delta T$ followed by holding it for $\Delta t$ at that temperature. The annealing was started at $200 \mathrm{~K}$ with $\Delta T=2 \mathrm{~K}$ and $\Delta t=2 \sim 32 \mathrm{~h}$ until the temperature reached $212 \mathrm{~K}$. In total, the annealing was continued for more than one month. The heat capacity of the $\mathrm{II}_{\mathrm{a}}$ sample showed the peak temperature at $226 \mathrm{~K}$, which is $17 \mathrm{~K}$ higher than that of the $\mathrm{II}_{\mathrm{r}}$ sample. At the same time, the entropy of transition increased to some extent. There must exist two kinds of ordering process in the racemic camphor, each possessing an essentially different relaxation time. The situation is interpreted in terms of the Gibbs energy curves for the phases III and II of D,L-camphor, as drawn schematically in Fig. 14. The point $G$ in this diagram represents a hypothetical transition point between phase III and II, both being fully ordered and disordered states with respect to their positional and orientational degrees of freedom. Both of the optical isomers in the racemic camphor are randomly located on the hexagonal lattice points in phase II. It is considered that orientational ordering takes place first on cooling the racemic camphor, leaving the chiral disorder behind. This chiral problem cannot appear in the lattice of D-camphor. Since D- and L-molecules have different conformations, molecular packing in the temporarily ordered state in the $\mathrm{II}_{\mathrm{r}}$ sample will not suitable to attain the ground state with complete order. Some molecules must have misorientations owing to the remaining positional disorder. Spatial rearrangement of both enantiomers requires a diffusional process, and therefore the chiral ordering occurs very slowly. The phase $\Pi_{r}$ irreversibly tends to stabilize to $\Pi_{r}^{\prime}, I I I_{r}{ }^{\prime \prime}, \cdots$ by the annealing process until it reaches ultimately $\mathrm{II}_{\mathrm{a}}$. Thus a countless number of metastable Gibbs energy curves corresponding to each stage of positional ordering are believed to exist in between.

In this way, the racemic camphor cooled in a normal way has two kinds of disorder, chiral disorder and partial orientational disorder coupled to the wrong positions of some molecules in the lattice. The latter would be the origin of the dielectric relaxation observed around $100 \mathrm{~K}$. Actually, the dielectric relaxation was found to decrease by the annealing. It is interesting to infer the structure of the fully 
ordered phase of racemic camphor. In contrast to the case of $\mathrm{Cs}_{\mathbf{x}} \mathrm{Tl}_{1-\mathrm{x}} \mathrm{NO}_{2}$ binary solutions, however, a superstructure model is suggested, because there were no indications of phase separation into each enantiomer crystals in the low-temperature phase of D,L-camphor. Structural determination of the well-aged racemic camphor would be worth pursuing.

\section{CONCLUDING REMARKS}

As stated by the third law of thermodynamics, all crystalline materials prefer ordered states at low temperatures. This statement is correct only when all the aspects of a system are in internal equilibrium. The "internal equilibrium" can be established in most cases within a reasonable time. Thus many phase transitions due to ordering of some aspects of a system have been observed with whole shape of the associated heat capacity anomaly. On the other hand, each degree of freedom has a characteristic relaxation time which changes rapidly with temperature. If the relaxation time becomes too long to establish the equilibrium, the associated physical quantities cannot follow the equilibrium values on cooling below a certain temperature which depends on the cooling rate.

In terms of Deborah number $D$, defined by Reiner (34) as a dimensionless quantity given by the ratio of relaxation time and observation time, the above situation can happen when the number $D$ becomes 1 . As far as $D \ll 1$, we can observe the whole shape of heat capacity anomaly associated with the ordering transition that can be determined by intermolecular or interionic interaction and by the cooperative nature of the interaction. Generally a relevant motion necessary for realizing an ordered state must overcome some potential barrier which is one important factor governing the relaxation time for the motion. Thus the condition $D=1$ will be encountered when the ordering transition occurs at low temperatures. This happened in ordinary ice crystal. A glass transition due to freezing out of reorientational motion of the water molecules was observed at around $100 \mathrm{~K}$ (35). The hypothetical equilibrium heat capacity was naturally hidden below that temperature. Absence of any ordering transitions that must remove the observed residual entropy (36) in ice has puzzled many scientists for more than fifty years. In this case, however, a small amount of particular impurity $(\mathrm{KOH})$ of the order of $10^{-4}$ mole fraction doped into the ice lattice dramatically accelerated the rate of motion and enabled us to remove the associated frozen-in entropy. The dopant induced a first-order transition at $72 \mathrm{~K}$ and disclosed the equilibrium heat capacity within our experimental time (37). The proton-ordered structure of the low-temperature phase was confirmed by a neutron diffraction method (38) on a heavy ice specimen doped with KOD, and designated as ice XI according to the order of discovery of many modifications. Increase in the thermal conductivity of ice by $17 \%$ at the transition $\mathrm{I}_{\mathrm{h}} \rightarrow \mathrm{XI}$ reflects increase in structural order of the low-temperature phase XI (39).

Similar dopant effects (40) were found in some clathrate hydrates which were otherwise in frozen disordered state with respect to two kinds of orientational degrees of freedom for the host water and guest molecules (41). The dopant again revealed first-order phase transitions that have so far been concealed for a kinetic reason (42). These experimental findings show us the important interplay between the phase and glass transitions in crystals, and also the wide validity of the third law of thermodynamics (43) whenever the kinetic hindrance is removed. At the same time, these studies open a new field of "doping chemistry". We cannot control the time, but we can control the molecular dynamics of disordered system by incorporating some particular kind of defects into the lattice of the system. The dopants might accelerate the molecular motion and release the system from immobilized frozen state to recover the equilibrium properties, as in the case of ordinary ice. Sometimes, the dopant will hinder an ordering transition to take place in our laboratory time, as in the case of thiophene crystal doped with benzene (44). Conversion of a non-equilibrium state to the equilibrium state or vice versa will be able to achieve by deep understanding of the roles played by any dopants. Thus, the correct recognition of the important interplay between the phase and glass transitions as well as the development of doping chemistry will surely enrich and enlarge the scope of material science.

\section{ACKNOWLEDGMENTS}

The experiments described here were carried out at Osaka University when the author was on its staff. The author would like to express his hearty thanks to many colleagues and collaborators for their enthusiastic collaboration and fruitful discussions. 


\section{REFERENCES}

1. J. Jäckel, Rep. Prog. Phys. 49, 171 (1985).

2. W. Kauzmann, Chem. Rev. 43, 219 (1948).

3. S. Brawer, Relaxation in Viscous Liquids and Glasses, p.37, Amer. Cer. Soc., Columbus (1985).

4. J. D. Mackenzie, Modern Aspects of Vitreous States, Vol. 3, p. 149, Butterworths, London (1964).

5. M. Oguni, H. Hikawa and H. Suga, Thermochim. Acta, 158, 143 (1990).

6. I. Tsukushi, O. Yamamuro and H. Suga, J. Therm. Anal. 37, 1359 (1991).

7. H. Suga and S. Seki, J. Non-cryst. Solids 15, 171 (1974) and Faraday Disc. No 69, p.21, Roy. Soc. Chem. (1980).

8. K. Mendelssohn, The World of Walther Nernst, The Rise and Fall of German Science, Macmillan, London, 1973.

9. H. Suga and T. Matsuo, Pure Appl. Chem. 61, 1123 (1987).

10. Y. Ogata, K. Kobayashi, T. Matsuo and H. Suga, J. Phys. E 17, 1054 (1984).

11. K. Kishimoto, H. Suga and S. Seki, Bull. Chem. Soc. Jpn. 53, 2748 (1980).

12. Y. Miyazaki, Doctoral Thesis, Osaka University, 1993.

13. T. Matsuo, H. Suga and S. Seki, Bull. Chem. Soc. Jpn. 41, 583 (1968).

14. H. Suga, T. Matsuo and S. Seki, Bull. Chem. Soc. Jpn. 38, 1115 (1965).

15. S. Haussühl, Solid State Commun. 13, 147 (1973).

16. M. D. Fountaine, C. R. Acad. Sci. Paris B281, 443 (1975)

17. M. Sugisaki, H. Suga and S. Seki, Bull. Chem. Soc. Jpn. 41, 1741 (1968).

18. T. Shimada, T. Matsuo, H. Suga and F. Luty, J. Chem. Phys. 85, 3530 (1986).

19. F. Luty, J. Ortiz-Lopez, Phys. Rev. Lett. 50, 1289 (1983).

20. C. Domb, Adv. Phys. 9, 1949 (1960) and G. A. Baker, Jr., Phys. Rev. 129, 99 (1963).

21. J. O. Clayton and W. F. Giauque, J. Amer. Chem. Soc. 54, 2610 (1932).

22. J. C. Burford and G. M. Graham, Can. J. Phys. 47, 4064 (1968).

23. E. K. Gill and J. A. Morrison, J. Chem. Phys. 45, 1585 (1966).

24. M. W. Melhuish and R. L. Scott, J. Phys. Chem. 68, 2301 (1964).

25. R. F. Curl, Jr., H. P. Hopkins, Jr. and K. S. Pitzer, J. Chem. Phys. 48, 4064 (1968).

26. T. Atake, H. Suga and H. Chihara, Chem. Lett. 1976, 567.

27. K. Moriya, T. Matsuo and H. Suga, J. Phys. Chem. Solids 44, 1103 (1983) and 44, 1121 (1983).

28. P. J. Protzenko and N. A. Brykova, Russ. J. Inorg. Chem. 10, 659 (1965)

29. J. Timmermans, J. Phys. Chem. Solids 18, 1 (1961).

30. K. L. Schäfer and U, Wagner, Z. Elektrochem. 62, 328 (1958)..

31. J. E. Anderson and W. P. Slichter, J. Chem. Phys. 41, 1922 (1958).

32. W. A. Yager and S. O. Morgan, J. Amer. Chem. Soc. 57, 2071 (1935).

33. T. Nagumo, T. Matsuo and H. Suga, Thermochim. Acta 139, 121 (1989).

34. M. Reiner, Phys. Today 17, 62 (1964)

35. O. Haida, T. Matsuo, H. Suga and S. Seki, J. Chem. Thermodyn. 6, 815 (1974).

36. W. F. Giauque and J. W. Stout, J. Amer. Chem. Soc. 58, 34 (1936).

37. Y. Tajima, T. Matsuo and H. Suga, Nature 299, 810 (1982).

38. A. J. Leadbetter, R. C. Ward, J. W. Clark, P. A. Tucker, T. Matsuo and H. Suga, J. Chem. Phys. 82, 424 (1982).

39. O. Andersson and H. Suga, Phys. Rev. B50, 6583 (1994).

40. O. Yamamuro and H. Suga, J. Therm. Anal. 35, 2025 (1989) and H. Suga, T. Matsuo and

O. Yamamuro, Supramol. Chem. 1, 221 (1993).

41. D. W. Davidson, Water - A Comprehensive Treatise, ed. by F. Franks, Vol. 2, P. 115, Plenum Press, New York (1984).

42. M. A. White and M. T. MacLean, J. Phys. Chem. 89, 1380 (1985).

43. J. Wilks, The Third Law of Thermodynamics, p. 40, Oxford Univ. Press, London (1961).

44. N. Okamoto, M. Oguni and H. Suga, Solid State Commun., 219 (1989). 